\title{
Kritische Bemerkungen zum Modell «Ärztenetzwerk mit Budgetverantwortung»
}

\author{
H. R. Baur
}

Die laufende Propaganda für diese Art Krankenversicherung, welche in letzter Zeit von vielen Politikern und auch von «MediX»-Ärzten betrieben wird, ist sehr einseitig. Die angepriesenen Kosteneinsparungen von 15 bis $25 \%$ sind in keiner Weise bewiesen. Dieses System wird seit Jahren in Teilen der Vereinigten Staaten angewandt und hat Tausende von frustrierten Patienten und auch Ärzten produziert. Weil die ärztliche Leistung pauschal abgegolten wird, besteht das Risiko, dass aus Kostengründen gewisse Untersuchungen und Therapien nicht durchgeführt werden. Patienten haben deswegen in den USA bereits Gerichtsklagen eingereicht und es sind mehrere Prozesse bekannt, welche wegen Todesfällen von Patienten geführt wurden, denen unter dem Managed-care-System medizinische Leistungen vorenthalten wurden. Viele Patienten haben sich von diesem System abgewandt, und nach Interventionen im amerikanischen Kongress mussten die Versicherungen ein Programm starten unter dem Titel «patients first», um das Vertrauen der Patientinnen und Patienten wieder zu gewinnen.

Nach vielen persönlichen Erfahrungen in Minnesota und San Diego, Hochburgen von Managed-care-Modellen, bin ich überzeugt, dass die Realität dieses Gesundheitssystems nicht dem Bild entspricht, welches in der Schweiz verbreitet wird.

Es ist schwierig zu verstehen, warum ein solches System, welches in der Praxis klare Fehler aufweist, in der Schweiz als wichtiger Fortschritt angepriesen wird. Wir sollten nicht die gleichen Fehler machen wie die Amerikaner, nur etwa 15 Jahre später! Die Befürworter behaupten, die Fehler dieses Systems könnten korrigiert werden. Dies ist kaum möglich, denn die fundamentalen Nachteile bleiben!

\section{Welches sind nun die fundamentalen Nachteile?}

Im konventionellen ärztlichen System ist der behandelnde Arzt der Advokat des Patienten. Er nimmt seine Rechte wahr und versucht, mit all seinem Wissen und Können die Gesundheit seines Patienten zu erhalten oder wiederherzustellen. Er setzt dabei alle notwendigen diagnostischen und therapeutischen Mittel ein, auch wenn manchmal der erwartete Erfolg als gering eingestuft werden muss. Sein wichtigstes Ziel ist die Gesundung des Patienten. Sein Honorar entspricht dem Umfang seiner Bemühungen.

Das System «Ärztenetzwerk mit Budgetverantwortung» entspricht klar dem System «capitation» der USA: die Versicherung zahlt dem Netzwerk pro Patient ein Kopfgeld. Es ist ihr Bestreben, dieses Kopfgeld möglichst tief zu halten, damit sie einen Gewinn erwirtschaften kann. Dem Gesundheitssystem wird dadurch bereits zu Beginn ein wesentlicher Teil der Mittel entzogen. In den USA sind mehrere Versicherungsgesellschaften auf diese Weise reich geworden, während sich die Ärztenetzwerke finanziell kaum behaupten konnten. Das ganze finanzielle Risiko liegt bei den Ärzten, die Versicherungen können mit risikolosen Einnahmequellen rechnen. Will die Schweiz diese Entwicklung wirklich kopieren?

Der entscheidende Unterschied zum traditionellen System besteht in der radikalen Umkehr der medizinischen Ethik: Während beim traditionellen System der Arzt für seine Bemühungen bezahlt wird, verdient er beim Netzwerksystem durch jeden Franken, den er dem Patienten vorenthält. Jede Untersuchung, jede Überweisung zum Spezialisten verursachen «Unkosten», die dem Netzwerkarzt direkt von seinem Verdienst abgezogen werden. Es versteht sich von selbst, dass auch idealistische Ärzte früher oder später in ein unüberwindliches ethisches Dilemma kommen, denn ihr Verdienst hängt letztlich davon ab, wie viele diagnostische und therapeutische Leistungen sie dem Patienten nicht angedeihen lassen. Aus finanziellen Gründen werden sie Untersuchungen weglassen und Therapien abkürzen, Sicherheitsstandards werden herabgesetzt. Sehr viele Hausärzte in der Schweiz können sich mit diesem System vor allem aus ethischen Gründen nicht identifizieren.

Der Einwand der Befürworter, dass der Patient selber merken wird, wenn er qualitativ nicht genügend versorgt wird, ist leider nicht zutreffend, da in sehr vielen Fällen der Patient 
nicht beurteilen kann, ob er medizinisch richtig und professionell auf hohem Niveau betreut wird. Er kann nicht abschätzen, welche Diagnostik und Therapie für seinen Gesundheitszustand notwendig sind.

Ärztenetzwerke können durchaus als Alternative auf dem Gesundheitsmarkt auftreten. Sie dürfen aber nicht durch drastische Massnahmen privilegiert behandelt werden, und es ist nicht annehmbar, wenn Ärzte keinen Kassenvertrag mehr bekommen und Patienten den doppelten Selbstbehalt bezahlen müssen, sofern sie sich nicht einem Netzwerk anschliessen.

Solche Massnahmen würden für viele Ärzte das Ende der freien Praxis und den Verlust ihrer finanziellen und beruflichen Unabhängigkeit bedeuten. Sie würden ihren Verdienst nur noch aus Kopfgeldern beziehen, deren Höhe abhängig wäre von der Ausgabefreudigkeit oder vom Sparwillen von vielen unbekannten Geschäftspartnern. Eine solch radikale Änderung in unserem seit Jahrzehnten bewährten Gesundheitssystem kann nicht ohne ausführliche Diskussion aller Beteiligten über Vor- und Nachteile eines solchen Systemwechsels erfolgen.

Einige wichtige weitere Nachteile dieses Systems:

1. Der Risikoausgleich mit Kopfprämien kann nur von einem grossen Netzwerk getragen werden (etwa 100000 bis 150000 Patienten). Es eignet sich nur für Agglomerationen. Der Hausarzt auf dem Lande kann kaum eingeschlossen werden. Es wäre äusserst asozial, wenn die Landbevölkerung mehr Selbstbehalt zahlen müsste, nur weil sie nicht in ein Ärztenetz eintreten kann.

2. Chronisch kranke und «teure» Patienten müssen durch komplizierte Rückversicherungen abgedeckt werden oder man wird versuchen, diese Patienten aus dem System zu nehmen und in eine medizinische Poliklinik abzuschieben.

\section{Zusammenfassung}

1. Das Ärztenetzwerksystem mit Budgetverantwortung kann als experimentelles System im schweizerischen Gesundheitsmarkt durchaus existieren. Es hat aber schwerwiegende Nachteile, die öffentlich diskutiert werden sollten.

2. Dieses System führt unweigerlich zu einem ärztlichen Dilemma, weil jede ärztliche Aufwendung das Einkommen des Arztes schmälert. Früher oder später werden wichtige Untersuchungen und Therapien zurückgehalten, was die Qualität der Betreuung entscheidend beeinflussen wird. Beispiele aus Amerika sind reichlich vorhanden. Das System ist asozial und benachteiligt die Landbevölkerung, wenn der Selbstbehalt bei Patienten ohne Netzwerk erhöht werden sollte.

3. Das System erteilt den Kassen eine zu grosse Macht. Sie werden Gelegenheit haben, ohne ein Risiko zu tragen, einen Teil der Prämien den Netzwerken vorzuenthalten und damit Gewinne zu erzielen. Diese Gewinne werden der Gesundheitsversorgung fehlen!

Die obigen Ausführungen stützen sich auf mehrjährige eigene Erfahrungen sowie Gespräche mit beteiligten Ärzten, Pflegedienstleiterinnen, Patientinnen und Patienten und Spitaladministratoren.

\section{Literaturauswahl}

1 Kassirer JP. Managed care and the morality of the marketplace. N Engl J Med 1995;333:50-2.

2 Angell M, Kassirer JP. Quality and the medical marketplace - following elephants. N Engl J Med 1996;335:883-5.

3 Bodenheimer T. The HMO backlash: righteous or reactionary? N Engl J Med 1996;335:1601-4.

4 Kassirer JP. Managing managed care's tarnished image. N Engl J Med 1997;337:338-9.

5 Miller TE. Managed care regulation: in the laboratory of the states. JAMA 1997;278:1102-9.

6 Kerr EA, Haxs RD et al. Primary care physicians' satisfaction with quality of care in California capitated medical groups. JAMA 1997;278:308-12.

7 Fye WB. Managed care and patients with cardiovascular disease. Circulation 1998;97:1895-6.

8 Davidoff $F$. Medicine and commerce. Is managed care a "monstrous hybrid"? Ann Intern Med 1998;128:496-9.

9 Davidoff F. Medicine and commerce. The gift. Ann Intern Med 1998;128:572-5.

10 Kassirer JP. Managed care - should we adopt a new ethic? N Engl J Med 1998;339:397-8.

11 Parmley WW. Introduction. $29^{\text {th }}$ Bethesda Conference Ethics in cardiovascular medicine (1997). JACC 1998;31:922-5.

12 Forrester JS., Kennedy JW. External influences on the practice of cardiology. $29^{\text {th }}$ Bethesda Conference Ethics in cardiovascular medicine (1997). JACC 1998;31:926-33.

13 Grumbach K, Osmond D et al. Primary care physicians' experience of financial incentives in managed-care systems. N Engl J Med 1998;339:1516-21.

14 Grumbach K. Primary care in the United States the best of times, the worst of times. N Engl J Med 1999;341:2008-10.

15 Sandy LG. Homeostasis without reserve - the risk of health system collapse. N Engl J Med 2002; 347:1971-5. 